\title{
An improved algorithm based on LEACH protocol
}

\author{
Wei Liu ${ }^{1} \quad$ LeLe Wang ${ }^{2}$ \\ ${ }^{1,2}$ College of Computer Science and Technology \\ Changchun University \\ ChangChun, 130022, P.R. China \\ e-mail: lw6803@163.com
}

\begin{abstract}
In this paper, taking into account the shortcomings of wireless sensor networks, LEACH algorithm, the algorithm has been improved on this basis. The design of the E-LEACH algorithm, which extend the network lifetime, improve node energy utilization, and the effectiveness of this algorithm through simulation experiments.
\end{abstract}

\section{Keywords-LEACH;sensor; routing protocols}

\section{INTRODUCTION}

In recent years, Wireless sensor networks came into being due to the rapid development of wireless technology, computer technology and sensor technology and Rapid integration. Wireless sensor network technology as a new type of network technology by researchers' widespread attention and extensive research.

There are also some disadvantages inherent in the sensor network, such as: energy low utilization, short life cycle, poor anti-interference ability. Good clustering algorithm can not only reduce the energy consumption of the sensor nodes, can also reduce communication interference, improve the efficiency of the MAC and routing protocols. Therefore, it is proposed that a highly efficient and stable rational algorithm has become an urgent need to solve the problem. In this paper, considering the residual energy for each node, a more efficient, more reasonably low overhead adaptive layered E-LEACH algorithm based on LEACH algorithm.

\section{WSN NODE STRUCTURE}

In wireless sensor networks, most important, the most fundamental component of the sensor nodes, these sensor nodes have low cost, small size, low power consumption and short-distance wireless communication characteristics. Monitoring of the sensing region, the wireless sensor node, simple processing, the data, and then sending or forwarding data, In addition, wireless sensor nodes need to consume a certain amount of energy itself. WSN there are a lot of different, have their own characteristics, but also a lot of the design and realization of the difficulties and problems, compared with the traditional wired network.

To act as nodes in WSN in three roles: the role of data acquisition, data transfer station or cluster head node. As data acquisition, data acquisition modules to collect the data of the surrounding environment, directly or indirectly through communication routing protocol to transfer the data to the remote base station; As a data relay station, the nodes in addition to the completion of the data acquisition task, but also receives the data of the neighbors will be forwarded to the distance of the base station closer to the neighbors or forwarded directly to the base station; As a cluster-head node, the node responsible for collecting the data collected for all nodes within the cluster, and by the fusion of the data sent to the base station. Be seen, the node has an important role in WSN.

\section{LEACH ALGORITHM ANALYSIS}

The basic idea of the LEACH protocol: "round " concept, The purpose of random selection of the cluster head node through each round of the cycle, and then rotate the cluster head node, so as to achieve balance and reduce energy consumption, extend the lifetime of the network. In LEACH protocol, each round consists of two parts. The first part is to build a cluster stage; the second part is stable session.

In practical wireless sensor network, after several rounds of post-election, the residual energy of each node will have a very different, near the cluster head node remaining energy away from the cluster head node residual energy. But traditional LEACH algorithm, all nodes become cluster head node probability is the same. Therefore, if selected after several rounds of data dissemination cluster head away from the base station node, the node's energy will soon be exhausted, and eventually became the failed node. Too many failed node will lead to paralysis of the entire network.

In addition, traditional LEACH algorithm, in every round of the initialization phase, the node to be added according to the strength of the broadcast signal of the cluster head section is received, select clusters. However, this solution is not always the optimal solution. Shown in Figure 1 is selected from the first cluster node, A, B, C, D is a base station, E non-first cluster node. Obviously, from the node E, $\mathrm{A}, \mathrm{C}$ the distance than the distance of the distance $\mathrm{B}$, however, if the $\mathrm{E}$ To the data transmitted to the base station $\mathrm{D}$, the optimal cluster head should be selected B. Because both election $\mathrm{C}$ or to vote for $\mathrm{A}$, energy consumption of the entire network is greater than $\mathrm{B}$ path. 


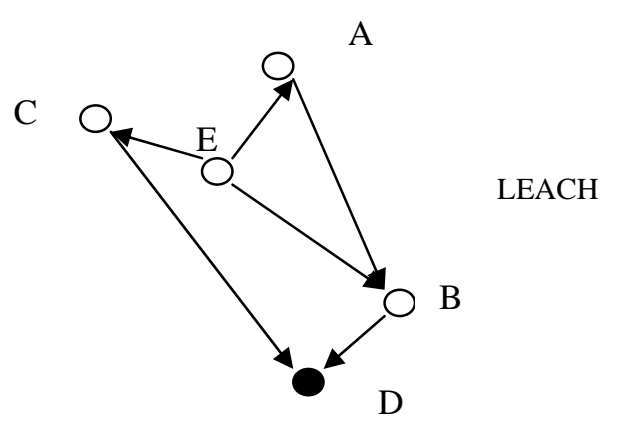

Figure 1 Node locations

\section{LEACH IMPROVED ALGORITHM}

A LEACH algorithm inadequacy, this article is designed E-LEACH algorithm to largely solve the above problems, E-LEACH algorithm build cluster after two choices. The first collection of nodes selected to meet the energy conditions and from the minimal condition, The second option is true cluster head selection phase, randomly selected to meet the requirements in the collection of the cluster head node.

First, E-LEACH algorithm introduces the concept of the energy threshold. Energy threshold is to determine whether the node can be used as a prerequisite of the cluster head node. Energy threshold of the formula:

$$
E(r)=K p E_{r} / m
$$

Where $\mathrm{E}(\mathrm{r})$ of the $\mathrm{r}$ - round of the energy threshold, $\mathrm{K}$ is an energy threshold factor; $p$ is the desired percentage of the share of all valid node cluster head node, $\mathrm{Er}$ is the sum of the energy of the randomly selected node r-cycle network, $\mathrm{m}$ is the total number of nodes of the cluster head round $r$. In before every a cluster head selection, compared to each meet the conditions of the cluster head node energy and energy threshold, Node energy is less than the energy threshold, the node removed from the cluster head node candidates.

Secondly, E-LEACH algorithm introduces a distance factor:

$$
w(i)=(d m-d(i)) / d m
$$

Wherein $\mathrm{dm}$ is the maximum distance of the node to the base station monitored area, $d$ (i) for the node $i$ to the distance of the base station. With the distance factor, in each round to select a cluster head node and the data sent to the base station, will take into account the distance cost. We use this method to select the path with the smallest data transmission distance.

After a first choice, we can get a cluster head candidate set Q.

$$
T(n)=\left\{\begin{array}{l}
1-p\left[r \bmod \left(\frac{1}{p}\right)\right], n \in Q \\
0,
\end{array}\right.
$$

$\mathrm{Q}$ is $1 / \mathrm{p}$ round did not become a cluster head and energy is greater than the energy threshold node collection.
Non-cluster head node joins the cluster, also with reference to the energy threshold and the distance factor, selected energy loss minimum transmission path.

\section{SimUlations AND EXPERIMENTAL ANALYSIS}

The program uses MATLAB simulation, $0.75 \mathrm{~K}$ simulations, and the sensor nodes are randomly distributed in the plane region of $1000 \times 1000$. 100 sensor nodes, each node initial energy $3 \mathrm{~J}$ base station coordinates $(50,750)$, the packet size is 20bytes. Figure 2 gives the LEACH algorithm E-LEACH algorithm survival number of nodes over time changes. Figure 3 shows the relationship between LEACH algorithm and E-LEACH algorithm transfer the amount of data and energy consumption.

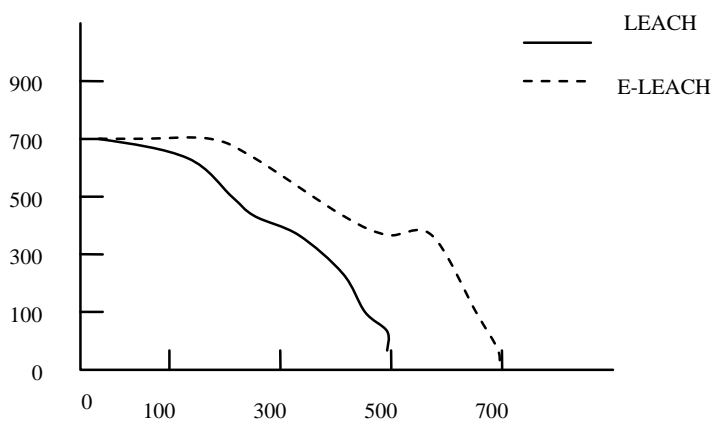

Figure 2 Surviving nodes

By the simulation of this experiment can be seen in the same conditions of the initial nodes, The LEACH algorithm of wireless sensor network nodes survive significantly lower than the E-LEACH algorithm for wireless sensor networks and under conditions to transfer the same amount of data, the less energy to be consumed by the E-leach algorithm.

\section{CONCLUSIONS}

In this paper, the LEACH algorithm based consider the residual energy of each node in each round inconsistencies , selected path is not the most energy-efficient path and proposed the concept of the energy threshold and distance factor, energy threshold and distance factors to determine the preferred cluster head collection selected on the basis of the cluster head node. Optimization algorithm selected by the head of such a cluster, and extends the network lifetime, improve node energy utilization. 


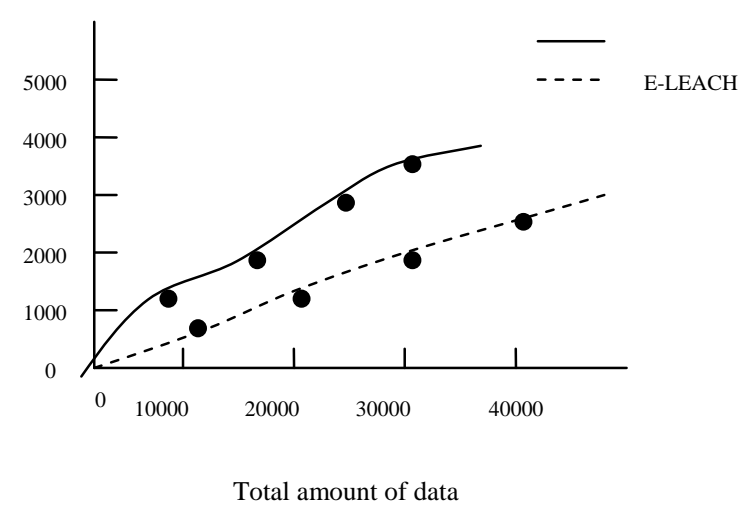

Figure 3 the amount of data and energy consumption relationship

\section{ACKNOWLEDGMENT}

This work is one part of the ChangChun International Science and Technology Cooperation Project (11GH11).

\section{REFERENCES}

[1]Li Jianzhong, Gao hong, Advances in wireless sensor network, Computer Research and Development,2008(01)

[2] Tang yong, Zhou mingtian ,Advances in wireless sensor network routing protocols, Journal of Software,2006(03)

[3] Pang Liaojun, Jiao Licheng, Design and Analysis of Secure Routing Protocol for Wireless Sensor Networks, Chinese Journal of Sensors and Actuators,2008(09)

[4] LI Ting, FENG Yong, Survey on secure routing research in wireless sensor networks, Application Research of Computers,2012(12)

[5] Wang Y.Topology control for wireless sensor networks[J].Wireless Sensor Networksand Applications, 2008: 113 - 147.

[6] Akyildiz I, Vuran M.Wireless sensor networks[M].Wiley Publishing, Inc 2010.

[7] Bortniko V, Chockler G, Roytman A, et al. Bulletin board: a scalable and robust eventually consistent shared memory

over a peer-to-peer overlay[J]. ACM SIGOPS Operating Systems Review, 2010,44(2): 64-70.

[8]M.Hamzeh,S.Arab,S.M.Fakhraie,C.Lucas.An Improvement on LEACH Algorithm with a Fuzzy Processor .IEICE 2008.

[9] Wei Bo Hu Han-ying Fu Wen.An Improved LEACH Protocol for Data Bring and Aggregation in wrelss Sensor Networks.IEEE computer society.2008.398-401. 